\title{
Multiple Use One-sided Hypotheses Testing in Univariate Linear Calibration
}

K. Krishnamoorthy ${ }^{a}$, Pandurang M. Kulkarni ${ }^{b}$ and Thomas Mathew ${ }^{c, *}$

${ }^{a}$ Department of Mathematics, University of Southwestern Louisiana, Lafayette, LA 70504, U.S.A.

${ }^{b}$ Department of Mathematics and Statistics, University of South Alabama, Mobile, AL 36688, U.S.A.

${ }^{c}$ Department of Mathematics and Statistics, University of Maryland, 1000 Hilltop Circle, Baltimore, MD 21250, U.S.A.

\begin{abstract}
Consider a normally distributed response variable, related to an explanatory variable through the simple linear regression model. Data obtained on the response variable, corresponding to known values of the explanatory variable (i.e., calibration data), are to be used for testing hypotheses concerning unknown values of the explanatory variable. We consider the problem of testing an unlimited sequence of one sided hypotheses concerning the explanatory variable, using the corresponding sequence of values of the response variable and the same set of calibration data. This is the situation of multiple use of the calibration data. The tests derived in this context are characterized by two types of uncertainties: one uncertainty associated with the sequence of values of the response variable, and a second uncertainty associated with the calibration data. We derive tests based on a condition that incorporates both of these uncertainties. The solution has practical applications in the decision limit problem. We illustrate our results using an example dealing with the estimation of blood alcohol concentration based on breath estimates of the alcohol concentration. In the example, the problem is to test if the unknown blood alcohol concentration of an individual exceeds a threshold that is safe for driving.
\end{abstract}

AMS Subject Classification: 62F03, 62J05

Key words: Calibration curve; Calibration data; Decision limit; Detection limit; Multiple use; Non-central t-distribution.

*Corresponding author. email: mathew@math.umbc.edu 


\section{Introduction}

The problems addressed in this article are in the set up of a linear regression model where normality is assumed for the dependent variable. Thus let $y_{1}, y_{2}, \ldots, y_{N}$ be $N$ independent observations on a response variable corresponding to the values $x_{1}, x_{2}, \ldots, x_{N}$, respectively, of an explanatory variable, where,

$$
y_{j} \sim N\left(\alpha+\beta x_{j}, \sigma^{2}\right),
$$

$j=1,2, \ldots, N$. The $x_{j}$ 's are assumed to be non-random quantities. Now let $y_{0}$ be another observation corresponding to an unknown value, say $\theta$, of the explanatory variable, where $y_{0}$ is independent of the $y_{j}$ 's in (1.1). Thus we have

$$
y_{0} \sim N\left(\alpha+\beta \theta, \sigma^{2}\right)
$$

The problem of calibration, or inverse regression, deals with statistical inference concerning $\theta$. The set of $y_{j}$ 's in (1.1), corresponding to the known values $x_{j}(j=1,2, \ldots, N)$, is referred to as the calibration data. The relationship between the $y_{j}$ 's and the corresponding $x_{j}$ 's is known as the calibration curve. We are thus dealing with the situation where the calibration curve is a straight line.

Most of the available literature on the calibration problem deals with point estimation of $\theta$, or the construction of confidence regions for $\theta$. The purpose of this article is to address some hypothesis testing problems concerning $\theta$. More specifically, we shall consider the following testing problems:

$$
\text { (i) } H_{0}: \theta \geq c \text { vs } H_{1}: \theta<c, \quad \text { (ii) } H_{0}: \theta \leq c \text { vs } H_{1}: \theta>c
$$

where $c$ is a known number, specified in advance. Our investigation of this problem was motivated by the following application.

\section{Estimation of blood alcohol concentration - an example}

This example deals with the calibration of breath estimates of blood alcohol concentration, based on the results of a laboratory test. Here, the $y_{j}$ 's are breath estimates of blood alcohol concentrations and the $x_{j}$ 's are the actual concentration of alcohol in the blood, obtained by a laboratory test. The relevant data, given in Section 3, are based on a study conducted at Acadiana Criminalstics Laboratory, New Iberia, Louisiana. It turns out that the model (1.1) provides an adequate fit for the data. We can use the models (1.1) and (1.2) in order to estimate an unknown blood alcohol concentration $\theta$, after obtaining the corresponding breath estimate $y_{0}$. Such an estimation is preferable to the actual laboratory determination of the blood alcohol concentration, since it is much easier and faster to obtain the breath estimates. In this context, we would also like to test whether an unknown blood alcohol concentration $\theta$ is less than or equal to 0.10 or if it exceeds 0.10 , since the legal maximum limit of blood alcohol concentration while driving is $0.10 \%$ in many states in the USA (in some states it is $0.08 \%$ ). This is precisely the testing problem (ii) in (1.3).

The hypothesis testing problems in (1.3) will also arise in the context of decision and detection limit problems in bioassays and chemical assays. Suppose we want to determine the concentration of a chemical in a sample where a direct determination of the concentration may 
be difficult or expensive. On the other hand, it may be easier to obtain an indirect measurement, for example, spectral measurements. First, several samples having known concentrations (the $x_{j}$ 's) are prepared, and the corresponding indirect measurements (the $y_{j}$ 's) are obtained. Assuming that the relationship (1.1) holds, we can use this data for statistical inference concerning an unknown concentration, say $\theta$, in a sample, after obtaining the corresponding indirect measurement $y_{0}$. In this context, the testing problems in (1.3) will arise if we want to verify whether the unknown concentration is above a threshold, or if it is below. This is precisely the decision limit problem. We refer to Currie (1988) for a detailed discussion of this problem as it arises in analytical chemistry. The testing problems (1.3) in the context of bioassays are addressed, for example, in Dunne (1995). A good discussion of decision and detection limits, along with environmental applications, can be found in Gibbons (1994, Chapter 5). Other examples and applications are given in a number of books and articles; see the book by Brown (1993) and the article by Osborne (1991) for a review.

For the models (1.1) and (1.2), appropriate tests for the hypotheses in (1.3) can be derived under two different situations. The first is when the calibration data are used only once for the purpose of testing hypothesis. In the context of the chemical example mentioned above, this means that the calibration data are collected and used to test hypothesis concerning an unknown concentration of a single sample. A more realistic scenario is one where the same set of calibration data are used repeatedly in order to test hypotheses concerning a sequence of $\theta$-values, one at a time, after observing the corresponding sequence of responses. This is the situation of multiple use of the calibration data. In other words, we have a sequence of independent responses $y_{0 i}$, corresponding to a sequence of $\theta$ values $\theta_{i}$, following the model

$$
y_{0 i} \sim N\left(\alpha+\beta \theta_{i}, \sigma^{2}\right)
$$

similar to $(1.2), i=1,2,3, \ldots$. Correspondingly, we have the following sequence of hypotheses to be tested.

$$
\text { (i) } H_{0 i}: \theta_{i} \geq c_{i} \text { vs } H_{1 i}: \theta_{i}<c_{i}, \quad \text { (ii) } H_{0 i}: \theta_{i} \leq c_{i} \text { vs } H_{1 i}: \theta_{i}>c_{i} \text {, }
$$

where the $c_{i}$ 's are known scalars. For instance, in Example 1, we want to verify if for every individual tested, the blood alcohol concentration is no more than 0.10 , or no more than 0.08 , where the value 0.10 or 0.08 is to be used depending on the legal maximum limit in a state. In other words, we have hypotheses of the type (ii) in (1.5), where the $c_{i}$ 's can take two different values, 0.10 and 0.08 . In the decision limit problem, we may be interested in testing if the concentration of a chemical or a pollutant in different samples exceeds a particular safety threshold. Such tests may have to be done a large number of times, as and when a sample is obtained. In other words, we need to test a sequence of hypotheses of the type (i) or (ii) in (1.5). It is also clear that in many applications, the $c_{i}$ 's in (1.5) will have a common value.

Tests that we shall derive for testing the sequence of hypotheses in (1.5) will have two types of uncertainty statements associated with them. One with respect to the sequence of responses $y_{0 i}$ in (1.4), and a second uncertainty statement with respect to the calibration data, i.e., the $y_{j}$ 's in (1.1). This is formally explained in Section 2. The distinction between single use and multiple use of the calibration data are also made for the construction of confidence regions. In fact confidence regions that involve multiple use of the calibration data are derived subject to two types of uncertainty statements, similar to those mentioned above. We refer to the recent 
article by Mee and Eberhardt (1996) for an excellent discussion on this. The results in Dunne (1995) deal with the set up of single use of the calibration data. In fact Dunne (1995) provides solutions to the testing problems in (1.3) in the single use situation. Consequently, in this article, we shall study the problem for the case of multiple use of the calibration data.

In the next section, we shall derive the tests. The two uncertainty statements, mentioned earlier, are explicitly stated and tests are derived subject to these. In our derivations, we have assumed that $\beta>0$ in (1.1). Essentially, this amounts to assuming that the sign of $\beta$ is known. This is clearly a reasonable assumption since the nature of the dependence of the response variable on the explanatory variable will be known in actual applications. In case $\beta<0$, we can assume that the slope in (1.1) is $-\beta$, after multiplying $y_{j}$ by -1 . In fact, the tests (i.e., decision limits) derived in Dunne (1995) use the fact that the sign of $\beta$ is known. In Section 3, we apply our results to the alcohol concentration data, mentioned in example 1. Some concluding remarks appear in Section 4.

\section{The Test}

Among the two hypotheses in (1.5), here we shall consider only the sequence (i). Once we derive tests for testing (i), similar results can be obtained for testing the sequence (ii) in (1.5).

Let $\hat{\alpha}$ and $\hat{\beta}$ denote the least squares estimators of $\alpha$ and $\beta$ based on the $y_{j}$ 's in (1.1), and let $\hat{\sigma}^{2}$ denote the unbiased estimator of $\sigma^{2}$ based on the residual sum of squares. Then

$$
\hat{\beta}=\frac{\sum_{j=1}^{N}\left(x_{j}-\bar{x}\right)\left(y_{j}-\bar{y}\right)}{\sum_{j=1}^{N}\left(x_{j}-\bar{x}\right)^{2}}, \quad \hat{\alpha}=\bar{y}-\hat{\beta} \bar{x}, \quad \text { and } \quad \hat{\sigma}^{2}=\frac{1}{N-2} \sum_{j=1}^{N}\left(y_{j}-\hat{\alpha}-\hat{\beta} x_{j}\right)^{2},
$$

where $\bar{y}$ and $\bar{x}$ denote the averages of the $y_{j}$ 's and the $x_{j}$ 's, respectively. As pointed out in the introduction, we assume that $\beta>0$.

\subsection{Motivation of the test statistic}

Suppose $\alpha, \beta$ and $\sigma^{2}$ are known. Then, from (1.2),

$$
\hat{\theta}=\frac{y_{0}-\alpha}{\beta} \sim N\left(\theta, \frac{\sigma^{2}}{\beta^{2}}\right)
$$

Hence, we shall reject $H_{0}: \theta \geq c$ in favor of $H_{1}: \theta<c$ if $\frac{\hat{\theta}-c}{\sigma / \beta}$ is small, or equivalently, if $\frac{y_{0}-\alpha-\beta c}{\sigma}$ is small. In order to arrive at the latter conclusion, we have used the expression for $\hat{\theta}$ (given above), along with our assumption $\beta>0$. In practice, usually $\alpha, \beta$ and $\sigma^{2}$ are unknown, and we shall replace them by their estimators. Thus let

$$
T=\frac{y_{0}-\hat{\alpha}-\hat{\beta} c}{\hat{\sigma}}
$$

Our test for $H_{0}: \theta \geq c$ will reject $H_{0}$ in favor of $H_{1}: \theta<c$ if $T$ is small. Hence, for testing the sequence of hypotheses (i) in (1.5), consider the test statistic

$$
T_{i}=\frac{y_{0 i}-\hat{\alpha}-\hat{\beta} c_{i}}{\hat{\sigma}}
$$



We shall reject $H_{0 i}: \theta_{i} \geq c_{i}$ in favor of $H_{1 i}: \theta_{i}<c_{i}$ if

$$
T_{i}<k_{i}
$$

$i=1,2,3, \ldots$, where the $k_{i}$ 's are to be determined. Note that the test statistic $T_{i}$ depends on the response $y_{0 i}$ and also on the calibration data $y_{j}, j=1,2, \ldots, N$, through $\hat{\alpha}, \hat{\beta}$ and $\hat{\sigma}$. Furthermore, (2.3) represents a sequence of rejection regions, for $i=1,2,3, \ldots$

\subsection{Criterion for the derivation of the test}

The derivation of our test amounts to the computation of the $k_{i}$ 's in (2.3). We shall now explain the condition to be satisfied by the $k_{i}$ 's. Since the calibration data will be used repeatedly for testing a sequence of hypotheses, consider the proportion of $H_{0 i}$ 's that are rejected, when they are true, conditionally given the calibration data, i.e., conditionally given $\hat{\alpha}, \hat{\beta}$ and $\hat{\sigma}$. In other words, conditionally given $\hat{\alpha}, \hat{\beta}$ and $\hat{\sigma}$, consider the proportion of times $T_{i}<k_{i}$, under $H_{0 i}$ : $\theta_{i} \geq c_{i}, i=1,2,3, \ldots$. The $k_{i}$ 's are to be determined so that with a high probability, the calibration data will guarantee that this proportion is small (i.e., less than a specified bound), for all $\theta_{i}$ 's under the corresponding null hypothesis $H_{0 i}$. Note that given $\hat{\alpha}, \hat{\beta}$ and $\hat{\sigma}$,

$$
T_{i} \sim N\left[\frac{\alpha-\hat{\alpha}+\beta \theta_{i}-\hat{\beta} c_{i}}{\hat{\sigma}}, \frac{\sigma^{2}}{\hat{\sigma}^{2}}\right] .
$$

Hence, conditionally given $\hat{\alpha}, \hat{\beta}$ and $\hat{\sigma}, T_{i}<k_{i}$ is equivalent to

$$
z_{0 i}<\left[k_{i}-\frac{\alpha-\hat{\alpha}+\beta \theta_{i}-\hat{\beta} c_{i}}{\hat{\sigma}}\right] \frac{\hat{\sigma}}{\sigma}
$$

where $z_{0 i} \sim N(0,1)$. We shall now give an expression for the proportion of $H_{0 i}$ that are rejected (i.e., proportion of times $T_{i}<k_{i}$ ), conditionally given $\hat{\alpha}, \hat{\beta}$ and $\hat{\sigma}$. Let

$$
\Delta_{i}= \begin{cases}1, & \text { if } T_{i}<k_{i} \\ 0, & \text { otherwise }\end{cases}
$$

Conditionally given $\hat{\alpha}, \hat{\beta}$ and $\hat{\sigma}$, the $\Delta_{i}$ 's are independent Bernoulli random variables with success probability, say $p_{i}$, given by

$$
p_{i}=P\left(T_{i}<k_{i} \mid \hat{\alpha}, \hat{\beta}, \hat{\sigma}\right)=\Phi\left\{k_{i} \frac{\hat{\sigma}}{\sigma}-\frac{\alpha-\hat{\alpha}+\beta \theta_{i}-\hat{\beta} c_{i}}{\sigma}\right\}
$$

where $\Phi$ denotes the standard normal cdf. In order to arrive at (2.6), we have used the fact that conditionally given $\hat{\alpha}, \hat{\beta}$ and $\hat{\sigma}, T_{i}<k_{i}$ is equivalent to (2.4). If a sequence of $n$ tests are carried out, then the proportion of times $T_{i}<k_{i}$ is $\frac{1}{n} \sum_{i=1}^{n} \Delta_{i}$. By the strong law of large numbers, we get

$$
\frac{1}{n} \sum_{i=1}^{n} \Delta_{i} \longrightarrow \frac{1}{n} \sum_{i=1}^{n} p_{i}
$$

as $n$ becomes large. In other words, conditionally given $\hat{\alpha}, \hat{\beta}$ and $\hat{\sigma}$, the proportion of $H_{0 i}$ that are rejected is $\frac{1}{n} \sum_{i=1}^{n} p_{i}$; when $n$ is large. We want this proportion to be small, with a high 
probability, for all $\theta_{i}$ under the corresponding null hypotheses $H_{0 i}: \theta_{i} \geq c_{i}, i=1,2,3, \ldots$ That is, the $k_{i}$ 's must satisfy

$$
P_{\hat{\alpha}, \hat{\beta}, \hat{\sigma}}\left[\frac{1}{n} \sum_{i=1}^{n} p_{i} \leq \epsilon\right]=\gamma
$$

for all $\theta_{i}$ under the corresponding null hypotheses $H_{0 i}: \theta_{i} \geq c_{i}, i=1,2,3, \ldots$, n, where $\epsilon$ is a specified bound (say, $\epsilon=0.05$ ) and $\gamma$ is the chosen probability (say, $\gamma=0.95$ ). Due to our assumption $\beta>0$, it follows from the expression for $p_{i}$ in (2.6) that $p_{i}$ is maximum under $H_{0 i}$ : $\theta_{i} \geq c_{i}$ when $\theta_{i}=c_{i}$. Hence (2.7) holds for all $\theta_{i}$ satisfying $\theta_{i} \geq c_{i}$ if and only if it holds for $\theta_{i}=c_{i}$. Using expression for $p_{i}$ in (2.6), we thus get the following condition to be satisfied by the $k_{i}$ 's:

$$
P_{\hat{\alpha}, \hat{\beta}, \hat{\sigma}}\left[\frac{1}{n} \sum_{i=1}^{n} \Phi\left\{k_{i} \frac{\hat{\sigma}}{\sigma}-\frac{\alpha-\hat{\alpha}+(\beta-\hat{\beta}) c_{i}}{\sigma}\right\} \leq \epsilon\right]=\gamma
$$

The condition (2.8) can be interpreted as the "size of the tests" being small, i.e., less than or equal to $\epsilon$, with probability $\gamma$. We note that (2.8) must actually hold for every $n$.

Thus the criterion to be used for the derivation of the test, i.e., the computation of the $k_{i}$ 's, is the condition (2.8), which must hold for all $n \geq 1$. As is clear from the derivation of (2.8), this condition reflects the fact that the same calibration data are used a large number of times for testing hypotheses. The fact that the same calibration data are used repeatedly is also the reason why we don't want to consider the unconditional distribution of the $T_{i}$ 's in $(2.2)$.

\subsection{Computation of the $k_{i}$ 's}

Note that

$$
\alpha-\hat{\alpha}+(\beta-\hat{\beta}) c_{i}=(\alpha+\beta \bar{x}-\bar{y})+(\beta-\hat{\beta})\left(c_{i}-\bar{x}\right) .
$$

Define

$$
\begin{aligned}
& z_{1}=\left[\sum_{j=1}^{N}\left(x_{j}-\bar{x}\right)^{2}\right]^{1 / 2}(\beta-\hat{\beta}) / \sigma \sim N(0,1), \quad z_{2}=\sqrt{N}(\bar{y}-\alpha-\beta \bar{x}) / \sigma \sim N(0,1), \\
& u^{2}=(N-2) \frac{\hat{\sigma}^{2}}{\sigma^{2}} \sim \chi_{N-2}^{2}, \quad \text { and } \quad c_{1 i}=\frac{\left(c_{i}-\bar{x}\right)}{\left[\sum_{j=1}^{N}\left(x_{j}-\bar{x}\right)^{2}\right]^{1 / 2}} .
\end{aligned}
$$

The random variables $z_{1}, z_{2}$ and $u$ in (2.9) are independently distributed, and $\chi_{r}^{2}$ denotes the central chisquare distribution with $r$ df. The $k_{i}$ that we compute will be a function of $c_{1 i}$ given in (2.9). Hence from now on we shall use the notation $k\left(c_{1 i}\right)$ instead of $k_{i}$. Using the quantities in $(2.9),(2.8)$ simplifies to

$$
P_{z_{1}, z_{2}, u}\left[\frac{1}{n} \sum_{i=1}^{n} \Phi\left\{k\left(c_{1 i}\right) \frac{u}{\sqrt{N-2}}+z_{1} c_{1 i}+\frac{z_{2}}{\sqrt{N}}\right\} \leq \epsilon\right]=\gamma .
$$

It appears difficult to obtain $k\left(c_{1 i}\right)$ explicitly, except in the special case when the $c_{1 i}$ 's are all equal. We shall first consider this special case. From the expression for $c_{1 i}$, given in (2.9), it follows that the $c_{1 i}$ 's are all equal if and only if the $c_{i}$ 's are all equal. As already pointed out, the $c_{i}$ 's will be equal in many practical applications; see the discussion following (1.5). When 
the $c_{i}$ 's are all equal, let $c$ denote the common value of the $c_{i}$ 's and let $c_{0}$ denote the common value of the $c_{1 i}$ 's. Hence, from (2.9),

$$
c_{0}=\frac{(c-\bar{x})}{\left[\sum_{j=1}^{N}\left(x_{j}-\bar{x}\right)^{2}\right]^{1 / 2}} .
$$

In this special case, $(2.10)$ simplifies to

$$
P_{z_{1}, z_{2}, u}\left[\Phi\left\{k\left(c_{0}\right) \frac{u}{\sqrt{N-2}}+z_{1} c_{0}+\frac{z_{2}}{\sqrt{N}}\right\} \leq \epsilon\right]=\gamma
$$

or, equivalently,

$$
P_{z_{1}, z_{2}, u}\left[k\left(c_{0}\right) \frac{u}{\sqrt{N-2}}+z_{1} c_{0}+\frac{z_{2}}{\sqrt{N}} \leq z(\epsilon)\right]=\gamma
$$

where $z(\epsilon)$ denotes the $100 \epsilon^{\text {th }}$ percentile of the standard normal distribution. We note that the condition (2.12), or equivalently, (2.13), is essentially the condition to be satisfied by one sided tolerance intervals. Indeed, we can arrive at (2.12) by starting with the expression for a one sided tolerance interval and using the fact that $\beta>0$. The solution to the one sided tolerance interval problem is already known; see Guttman $\left(1970\right.$, pp. 87-89). The solution to $k\left(c_{0}\right)$ is given by

$$
k\left(c_{0}\right)=\left(\frac{1}{N}+c_{0}^{2}\right)^{1 / 2} t_{1-\gamma}\left[N-2 ; \frac{z(\epsilon)}{\left(\frac{1}{N}+c_{0}^{2}\right)^{1 / 2}}\right],
$$

where $t_{1-\gamma}[r ; \lambda]$ denotes the $100(1-\gamma)^{\text {th }}$ percentile of the noncentral t-distribution with $\mathrm{df}=r$ and noncentrality parameter $\lambda$. Thus in the special case where the $c_{i}$ 's are all equal to a common value $c, k\left(c_{0}\right)$ in (2.14) satisfies (2.12), where $c_{0}$ is given in (2.11).

When the $c_{1 i}$ 's are not all equal, it is not possible to obtain $k\left(c_{1 i}\right)$ explicitly. In what follows, we shall explore the following possibility. Let $k\left(c_{1 i}\right)$ satisfy

$$
P_{z_{1}, z_{2}, u}\left[\Phi\left\{k\left(c_{1 i}\right) \frac{u}{\sqrt{N-2}}+z_{1} c_{1 i}+\frac{z_{2}}{\sqrt{N}}\right\} \leq \epsilon\right]=\gamma
$$

In other words,

$$
k\left(c_{1 i}\right)=\left(\frac{1}{N}+c_{1 i}^{2}\right)^{1 / 2} t_{1-\gamma}\left[N-2 ; \frac{z(\epsilon)}{\left(\frac{1}{N}+c_{1 i}^{2}\right)^{1 / 2}}\right] .
$$

The expression in (2.16) follows since (2.15) is similar to (2.12). We shall numerically investigate whether $k\left(c_{1 i}\right)$ in (2.16) will satisfy $(2.10)$ for various sequences $\left\{c_{1 i}\right\}$. Note that (2.15) is a tolerance interval condition and is quite different from (2.10). In a recent article on the construction of confidence regions in the calibration problem. Mee and Eberhardt (1996) have indicated that intervals obtained using a tolerance interval condition may satisfy the requirements of multiple use confidence regions. Motivated by this, we shall explore whether $k\left(c_{1 i}\right)$ in (2.16) will satisfy $(2.10)$.

\subsection{Numerical results for $k\left(c_{1 i}\right)$ in (2.16)}

We have carried out some numerical results to check if $k\left(c_{1 i}\right)$ in $(2.16)$ will satisfy (2.10). For this purpose, we need to simulate the left hand side (lhs) of (2.10) using $k\left(c_{1 i}\right)$ given in (2.16), 
for various sequences $\left\{c_{1 i}\right\}$. Note that when the $c_{1 i}$ 's are all equal, i.e., when the $c_{i}$ 's are all equal, such a numerical study is not necessary, since, in this case, $k\left(c_{0}\right)$ satisfies $(2.12)$, where $c_{0}$ denotes the common value of the $c_{1 i}$ 's. Thus the numerical results that we report here are only for the case when the $c_{1 i}$ 's are unequal. We shall first discuss our choices for the sequence $\left\{c_{1 i}\right\}$. When the calibration data are collected based on a carefully designed experiment, then the $x_{j}$ 's in (1.1) will cover the range of practical interest. Hence it is reasonable to assume that the $\theta_{i}$ 's in (1.4) belong to the range determined by the $x_{j}$ 's, i.e.,

$$
\min \left(x_{j}\right) \leq \theta_{i} \leq \max \left(x_{j}\right)
$$

$i=1,2,3, \ldots$ Consequently, when (2.17) holds, the $c_{i}$ 's in (1.5) should also belong to the range of the $x_{j}$ 's. In other words, when we test the null hypothesis $H_{0 i}: \theta_{i} \geq c_{i}$, we are testing if $\theta_{i}$ is greater than or equal to a specified value in the range of the $x_{j}$ 's. Hence we shall assume

$$
\min \left(x_{j}\right) \leq c_{i} \leq \max \left(x_{j}\right)
$$

$i=1,2,3, \ldots$ From the definition of $c_{1 i}$ given in $(2.9),(2.18)$ gives

$$
\frac{\min \left(x_{j}\right)-\bar{x}}{\left[\sum_{j=1}^{N}\left(x_{j}-\bar{x}\right)^{2}\right]^{1 / 2}} \leq c_{1 i} \leq \frac{\max \left(x_{j}\right)-\bar{x}}{\left[\sum_{j=1}^{N}\left(x_{j}-\bar{x}\right)^{2}\right]^{1 / 2}}
$$

$i=1,2,3, \ldots$. In particular, (2.19) implies that

$$
-1 \leq c_{1 i} \leq 1
$$

The actual bounds for $c_{1 i}$, given in (2.19), could be much narrower than $[-1,1]$. In any case, in order to investigate whether $k\left(c_{1 i}\right)$ in $(2.16)$ will satisfy $(2.10)$, it appears reasonable to restrict $c_{1 i}$ 's in the interval $[-1,1]$. In our numerical results, we have also considered the wider interval $[-2,2]$.

For various sequences $\left\{c_{1 i}\right\}, i=1,2, \ldots, 10,000$, we have simulated the following quantity.

$$
P_{z_{1}, z_{2}, u}\left[\frac{1}{10,000} \sum_{i=1}^{10,000} \Phi\left\{k\left(c_{1 i}\right) \frac{u}{\sqrt{N-2}}+z_{1} c_{1 i}+\frac{z_{2}}{\sqrt{N}}\right\} \leq \epsilon\right],
$$

based on 10,000 simulations. The IMSL Fortran function subroutine TNIN is used to compute $k\left(c_{1 i}\right)$. Note that $(2.21)$ is an approximation to the lhs of $(2.10)$. Since $(2.10)$ holds exactly when the $c_{1 i}$ 's are all equal, $(2.10)$ is expected to hold approximately when the $c_{1 i}$ 's are nearly equal. Thus it is necessary to investigate whether (2.10) will hold when the $c_{1 i}$ 's are as unequal as possible. In any finite sequence $\left\{c_{1 i}\right\}$, with $c_{1 i} \in[-a, a]$, the $c_{1 i}$ 's are as unequal as possible, i.e., the variance among them is a maximum, when half of the $c_{1 i}$ 's are equal to $-a$ and the remaining half are equal to $a$. In this case, (2.21) reduces to

$$
P_{z_{1}, z_{2}, u}\left[\frac{1}{2}\left(\Phi\left\{k(-a) \frac{u}{\sqrt{N-2}}-z_{1} a+\frac{z_{2}}{\sqrt{N}}\right\}+\Phi\left\{k(a) \frac{u}{\sqrt{N-2}}+z_{1} a+\frac{z_{2}}{\sqrt{N}}\right\}\right) \leq \epsilon\right] .
$$

The following IMSL subroutines are used in the numerical studies: TNIN to compute $k\left(c_{1 i}\right)$; RNNOA to generate normal variates; and RNCHI to generate chi-square variates. For $N=20$, $\epsilon=0.05$ and $0.01, \gamma=0.99,0.95,0.90$ and 0.85 , and $a=2,1,0.5$ and 0.1 , Table 1 gives the 
simulated values of (2.22), based on 10,000 simulations. When the sequence $\left\{c_{1 i}\right\}, i=1,2, \ldots$, 10,000 consists of 10,000 equispaced values in the interval $[-2,2]$ and $[-1,1]$, the simulated values of (2.21) appear in Table 2. If $10,000 c_{1 i}$ 's are randomly generated according to a standard normal distribution, the simulated values of (2.21) appear in Table 3.

Table $1 a$

The simulated values of (2.22) for $N=20$ and $\epsilon=0.05$, for different values of $\gamma$ and $a$

\begin{tabular}{ccccc}
\hline$a$ & & $\gamma$ & & \\
& 0.99 & 0.95 & 0.90 & 0.85 \\
\hline 2 & 0.987 & 0.936 & 0.852 & 0.777 \\
1 & 0.991 & 0.950 & 0.889 & 0.826 \\
0.5 & 0.993 & 0.959 & 0.916 & 0.857 \\
0.1 & 0.990 & 0.952 & 0.902 & 0.849 \\
\hline
\end{tabular}

Table $1 b$

The simulated values of (2.22) for $N=20$ and $\epsilon=0.01$, for different values of $\gamma$ and $a$

\begin{tabular}{ccccc}
\hline$a$ & \multicolumn{4}{c}{$\gamma$} \\
& 0.99 & 0.95 & 0.90 & 0.85 \\
\hline 2 & 0.985 & 0.923 & 0.848 & 0.753 \\
1 & 0.991 & 0.938 & 0.865 & 0.799 \\
0.5 & 0.991 & 0.952 & 0.885 & 0.824 \\
0.1 & 0.992 & 0.949 & 0.897 & 0.857 \\
\hline
\end{tabular}

Table 2

The simulated probabilities (2.21) for $N=20$ when the sequence $\left\{c_{1 i}\right\}$ assumes 10,000 equispaced values in the interval $[-a, a]$

\begin{tabular}{cccccc}
\hline$[-a, a]$ & & \multicolumn{5}{c}{} \\
& $\epsilon$ & 0.99 & 0.95 & 0.90 & 0.85 \\
\hline$[-2,2]$ & 0.05 & 0.989 & 0.954 & 0.902 & 0.852 \\
{$[-1,1]$} & 0.05 & 0.992 & 0.951 & 0.898 & 0.851 \\
{$[-2,2]$} & 0.01 & 0.991 & 0.954 & 0.907 & 0.846 \\
{$[-1,1]$} & 0.01 & 0.993 & 0.947 & 0.902 & 0.851 \\
\hline
\end{tabular}

Table 3

The simulated probabilities $(2.21)$ for $N=20$ when the sequence $\left\{c_{1 i}\right\}$ is generated based on $N(0,1), i=1,2, \ldots, 10,000$

\begin{tabular}{ccccc}
\hline & \multicolumn{4}{c}{} \\
$\epsilon$ & 0.99 & 0.95 & 0.90 & 0.85 \\
\hline 0.05 & 0.989 & 0.950 & 0.897 & 0.832 \\
0.01 & 0.992 & 0.951 & 0.899 & 0.834 \\
\hline
\end{tabular}

The simulation results indicate that $k\left(c_{1 i}\right)$ in (2.16) will satisfy (2.10) reasonably well at least in situations where the range for $c_{1 i}$, given in (2.19), is a narrow interval. As expected, (2.10) is 
more satisfactorily met for narrower intervals. As already pointed out, it appears quite reasonable to assume that $-1 \leq c_{1 i} \leq 1$. In practice, the range for $c_{1 i}$ is likely to be much narrower, especially when $N$ is somewhat large. This should be clear from (2.19). In terms of meeting the condition (2.10), the results are somewhat unsatisfactory in the set up of Table 1, especially for $\gamma=0.85$. However, Table 1 represents the 'worst case scenario', since (2.22) corresponds to the situation where half of the $c_{1 i}$ 's are equal to $-a$ and the remaining half are equal to $a$ (for $a=2,1,0.5,0.1$ ). In other words, among the sequence of hypotheses being tested, half of the time we are testing if the parameter is greater than or equal to one extreme of the parameter space (i.e., $-a$ ) and the other half deals with testing if the parameter is greater than or equal to the other extreme (i.e., a). In actual applications, this appears unrealistic. Nevertheless, the purpose of reporting the numerical results in Table 1 is to see of (2.10) (i.e, (2.22)) is met in this extreme situation. For large values of $\gamma$ the results are quite satisfactory, especially when $a$ is small. In any case, the numerical results in Table 1-Table 3 provide evidence regarding the extent to which (2.16) will satisfy (2.10). The overall conclusion is that in practical applications that call for multiple use of the calibration data for testing the hypotheses in $(1.5), k\left(c_{1 i}\right)$ given in (2.16) is quite adequate for meeting the requirement (2.10).

\subsection{Testing the hypothesis 1.5 (ii)}

Tests for the hypotheses (ii) in (1.5) can be similarly derived. The rejection regions now take the form $T_{i}>k_{1}\left(c_{1 i}\right)$, where $T_{i}$ is given in (2.2) and $c_{1 i}$ is given in (2.9). When the $c_{1 i}$ 's are all equal to $c_{0}, k_{1}\left(c_{0}\right)$ is given by

$$
k_{1}\left(c_{0}\right)=\left(\frac{1}{N}+c_{0}^{2}\right)^{1 / 2} t_{\gamma}\left[N-2 ; \frac{z(1-\epsilon)}{\left(\frac{1}{N}+c_{0}^{2}\right)^{1 / 2}}\right],
$$

similar to (2.14). Also, $k_{1}\left(c_{1 i}\right)$ has an expression similar to that of $k\left(c_{1 i}\right)$ in (2.16).

\section{An Example}

A study was conducted at Acadiana Criminalstics Laboratory, New Iberia, Louisiana, to compare the breath estimates of blood alcohol concentration with those determined by a laboratory test. A sample of 15 subjects was used. In Table 4, we present the breath estimates $\left(y_{j}\right)$ obtained using Breathalyzer Model 5000 and the results of the laboratory test $\left(x_{j}\right)$. These numbers are percentages of alcohol concentration in blood. Here we assume that the $x_{j}$ 's are accurately measured, i.e., the measurement errors are small enough to be ignored, so that the $x_{j}$ 's can be assumed to be fixed. A simple linear regression model was fit with $y$ regressed on $x$ based on all 15 pairs of observations. The fitted model is $y=0.00135+0.958 x$ and the value of $R^{2}$ is 0.93 . The normal probability plot of the residuals is reasonably linear and hence the distribution of error terms does not depart from a normal distribution. Since in many states in the USA the legal maximum limit of blood alcohol concentration while driving is $0.1 \%$, we want to test whether $\theta \leq 0.10$ or $\theta>0.10$. In other words, here we shall consider the situation where the $c_{i}$ 's in (1.5) are all equal and equal to 0.10 . In this problem, one may want to control the probability of concluding $\theta>0.10$ when in fact $\theta \leq 0.10$. Thus we shall consider the following null and alternative hypotheses.

$$
H_{0}: \theta \leq 0.10 \quad \text { vs } \quad H_{1}: \theta>0.10 \text {. }
$$


We shall carry out the test using $\epsilon=0.05$ and $\gamma=0.95$. From (2.23),

$$
k_{1}\left(c_{0}\right)=\left(\frac{1}{N}+c_{0}^{2}\right)^{1 / 2} t_{0.95}\left[N-2 ; \frac{z(0.95)}{\left(\frac{1}{14}+c_{0}^{2}\right)^{1 / 2}}\right] .
$$

We reject $H_{0}$ for the $i^{\text {th }}$ individual if $T_{i}>k_{1}\left(c_{0}\right)$.

In Table 4, we give the classical estimates obtained by solving from the fitted model for $x$, and the values of test statistics $T_{i}$. The $T_{i}$ 's are computed using the leave-one-out method; for example to compute the point estimate and the value of the test statistic corresponding to $y_{1}=$ 0.145 in Table 4 , the observation $(.145,160)$ is not used to fit the model. Thus, $N=14$, in $(3.2)$. Fitting the model by deleting the first observation, we get $\hat{\alpha}=.0007, \hat{\beta}=.97, c_{0}=-0.077$, $T_{i}=3.40$, and $k_{1}\left(c_{0}\right)=2.67$. Furthermore, $\hat{x}_{1}=\frac{y_{1}-\hat{\alpha}}{\hat{\beta}}=\frac{.145-.0007}{.97}=.149$. This process can be carried out by deleting each observation in Table 4 .

Table 4

Blood Alcohol Concentrations Data, Estimates and Test Statistics

\begin{tabular}{cccccc}
\hline & $y_{i}$ & $x_{i}$ & $\hat{x}_{i}$ & $T_{i}$ & $k_{1}\left(c_{0}\right)$ \\
\hline 1 & .145 & .160 & .149 & 3.40 & 2.67 \\
2 & .156 & .170 & .160 & 4.18 & 2.67 \\
3 & .181 & .180 & .189 & 6.00 & 2.67 \\
4 & .108 & .100 & .112 & 0.85 & 2.68 \\
5 & .180 & .170 & .189 & 6.27 & 2.67 \\
6 & .112 & .100 & .117 & 1.19 & 2.68 \\
7 & .081 & .060 & .086 & -1.11 & 2.69 \\
8 & .104 & .100 & .108 & 0.53 & 2.68 \\
9 & .176 & .170 & .185 & 5.78 & 2.67 \\
10 & .048 & .056 & .047 & -3.56 & 2.69 \\
11 & .092 & .111 & .093 & -0.47 & 2.68 \\
12 & .144 & .162 & .147 & 3.38 & 2.67 \\
13 & .121 & .143 & .124 & 1.73 & 2.67 \\
14 & .065 & .079 & .065 & -2.42 & 2.68 \\
15 & .000 & .006 & -.006 & -7.05 & 2.71 \\
\hline
\end{tabular}

We observe from Table 4 that the test results are in agreement with those based on laboratory test except for the 11 th and 13 th subjects. We also note that for the 15 th observation, $x_{i}$ $=0.006$ and $\hat{x}_{i}=-0.006$. This discrepancy may be due to the fact that the corresponding $y_{i}$ $=0$ and $x_{i}$ is very close to zero.

\section{Concluding Remarks}

In this article, we have investigated hypothesis testing in the calibration problem. In the univariate case, we have succeeded in deriving appropriate tests for testing one sided hypotheses in the situation of multiple use of the calibration data. The results are illustrated using an example. The two sided testing problem is not considered in this article, since tests in this 
context can be obtained by inverting the multiple use confidence regions that are available in the literature. One-sided multiple use confidence regions can certainly be developed and can be used for testing the hypotheses in (1.5). The simultaneous tolerance intervals in Mee, Eberhardt and Reeve (1991) can be used to obtain conservative multiple use confidence regions, and hence conservative multiple use tests, for the two sided testing problem. The one-sided simultaneous tolerance intervals in Odeh and Mee (1990) can similarly be used for obtaining conservative multiple use tests for the one sided testing problems in (1.5). However, in the present article, our goal has been to investigate whether the tolerance interval condition can be used for carrying out multiple use hypotheses tests. The numerical results in Section 3 show that this is indeed the case. Note that our results are applicable only in the univariate set up and similar results are currently not available in the multivariate case. The problem of testing hypothesis in the multivariate calibration problem is currently under investigation.

\section{Acknowledgement}

Mathew's research was supported in part by National Science Foundation Grant DMS 95-30932. Kulkarni's research was supported in part by NASA-JOVE grant. The authors are thankful to Denise Benton for providing useful references related to the example in Section 5 and helping us to get the data, and to Kevin Ardoin for providing the data. They also wish to thank the reviewers for many helpful comments, and for drawing attention to the literature on detection limits.

\section{References}

Brown, P. J. (1993). Measurement, Regression and Calibration. Oxford University Press, New York.

Currie, L. A. (1988). Detection in Analytical Chemistry: Importance, Theory and Practice. American Chemical Society, Washington, DC.

Dunne, A. (1995). Decision and detection limits for linear homoscedastic assays. Statist. Medicine 14, 1949-1959.

Gibbons, R. D. (1994). Statistical Methods for Groundwater Monitoring. John Wiley, New York.

Guttman, I. (1970). Statistical Tolerance Regions, Classical and Bayesian. Charles Griffin \& Co. Ltd., London.

Mee, R. W. and K. R. Eberhardt (1996). A Comparison of uncertainty criteria for calibration. Technometrics 38, 221-229.

Mee, R. W., Eberhardt, K. R. and C. P. Reeve (1991). Calibration and simultaneous tolerance intervals for regression. Technometrics, 33, 211-219.

Odeh, R. E. and R. W. Mee (1990). One-sided simultaneous tolerance limit for regression. Comm. Statist. Simulation and Computation, 19, 663-680.

Osborne, C. (1991). Statistical calibration: a review. Internat. Statist. Rev.. 59, 309-336. 